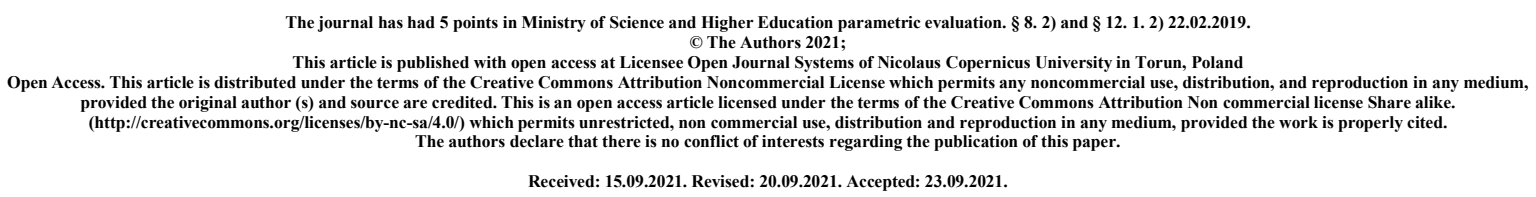

\title{
Positive and negative aspects of sauna bathing - current knowledge status
}

\author{
Mateusz Sobczyk $^{1}$, Paulina Oleksa ${ }^{1}$, Piotr Wójcik ${ }^{1}$, Daria Żuraw ${ }^{1}$, Monika Rogowska ${ }^{1}$, \\ Małgorzata Słaboń ${ }^{1}$
}

${ }^{1}$ Student Research Circle at the Department of Epidemiology and Clinical Research Methodology, Medical University of Lublin, Poland

\begin{abstract}
Introduction: Sauna bathing as a health-promoting activity has been known to mankind for millennia. Sauna session, which consists of alternate overheating and then rapidly cooling the body, has a beneficial effect on the health of the body confirmed by scientific research. In the last few decades, sauna bathing has become more available due to the growing market of recreational services such as water parks and sauna parks. Regular use of sauna bathing reduces the risk of cardiovascular diseases, musculoskeletal disorders, mental stress, accelerates the process of regeneration after physical exertion, as well as increases resistance to the harmful effects of certain environmental factors. Despite the health benefits known for many years, the physiological mechanisms occurring in the body during sauna sessions still remain unknown. Sauna bathing treatments can be an effective complement to the process of treatment and rehabilitation of patients with cardiovascular diseases, musculoskeletal diseases, respiratory tract diseases, skin diseases and many others.
\end{abstract}

Material and methods: A literature analysis on sauna bathing was carried out within the Pubmed and Google scholar platforms. The following keywords were used in search: sauna bathing, infrared sauna, cardiovascular diseases.

Purpose of the work: The aim of the following analysis is to present a historical outline of the use of sauna bathing, the health aspect, risks and the assumptions and indicating an important need for further research into the effects of sauna bathing on the body.

Key words: Sauna bathing, Infrared sauna, health training, prevention of civilisation diseases

1. Introduction, history of sauna bathing and aim of the work 
According to historical sources, the custom of sauna bathing originated in central Asia. The customs of using installations resembling today's saunas occurred in the areas of Syria, Egypt, Greece and the areas inhabited by the Slavic peoples. In the 1st century BC, the custom of using saunas reached Rome, where the so-called Roman thermae were created. In countries inhabited by Muslims, Turkish baths were spread, which began to be established in the 3rd century AD. Turkish baths are a combination of Syrian baths and Roman baths.

The country where sauna bathing is most popular is Finland. Finnish sauna bathing has been used for the purposes of pleasure, wellness, and relaxation. It is a tradition embedded in the culture of Finland, and it is basically accessible to everyone [1]. In Finland, 90\% of houses are equipped with saunas, so statistically there is one sauna for every four people. The tradition of using saunas has been known in this area for 2,000 years. In Finnish culture, when building a household, it is customary to start by building a sauna. For this reason, most of the scientific publications on the health benefits of sauna bathing are authored by Finnish scientists.

In medicine and sport, the sauna has played and continues to play a very important role. Although its effect on the body is relatively well understood, it is not fully explored. Research studies suggests that regular sauna use can reduce and prevent the risk of both acute and chronic diseases [1].

\section{Types of saunas}

There are many different forms of passive heat therapy (eg, traditional dry (finish) sauna, repeated hot water immersion, infrared sauna, Waon therapy, and Turkish bath) [1]. Everyone can choose the type of sauna according to their own tastes and medical indications [2]. A sauna is a room inside of which there is a heater, which allows to achieve a dry sauna (Finish also known as Swedish). During such a sauna bath there is a very high temperature of $80-100^{\circ} \mathrm{C}$ and the humidity level is kept at a very low level of about $10-20 \%$, which is much lower than in living rooms, where it is $45-65 \%$ [3]. Sauna sessions consist of several short stays in the sauna lasting from 5 to 20 minutes, interrupted by cooling down with cold water in a shower or bath [3]. The temperature in a steam sauna is about $70-90^{\circ} \mathrm{C}$, with relative humidity of about $25-39 \%$, which is achieved by pouring water over heated granite stones. The steam and herb sauna is suitable for more number of people due to its lower temperature of $45-65^{\circ} \mathrm{C}$. The humidity level is $40-65 \%$. There is a steam generator, which is passed through the herbs or aromatic oils, so that the human body takes an additional inhalation [2]. Turkish bath is based on maintaining the temperature in the range of $40-50^{\circ} \mathrm{C}$ along with high air humidity. Waon therapy, known in Japan, involves heating the body in a dry environment to about $60^{\circ} \mathrm{C}$ for about 15 minutes followed by a rest of about 30 minutes without previous rapid cooling of the body [3]. Another type is the infrared sauna, which is also built of wood, but the traditional heater is replaced by infrared emitters emitting FIR (far-infrared rays) [4]. The temperature is maintained within the range of $40-50^{\circ} \mathrm{C}$ without the addition of steam. Infrared radiation during the treatment is characterised by the fact that it penetrates deep into the tissues, even up to $0.5-3 \mathrm{~mm}$. This sauna is a great substitute for the traditional sauna due to the shorter time spent in the sauna, lesser influence on the heart rate and blood pressure and also lower costs of the therapy. Due to a different physical mechanism of heating the body, only $20 \%$ of the energy is spent on heating the room and as much as $80 \%$ on heating the body, which means that a stay in the sauna of 5 to 10 minutes is sufficient [2,5]. This makes the bath better tolerated by the patient and it is often used during the warm-up by athletes. Most scientific publications deal with the health effects of traditional Finnish/Swedish saunas, and therefore they will receive special attention in this review. 


\section{Influence of sauna bathing on physiological processes of human body}

Bathing in a dry (Finnish) sauna lasting up to 20 minutes leads to an increase in body temperature to about $39^{\circ} \mathrm{C}$. During the stay in the sauna, an average person excretes about 0.5 litres of water through sweating [3]. In young healthy people, during the stay in the sauna, the systolic blood pressure remains unchanged or temporarily increases, while the diastolic pressure decreases [6]. In patients treated for hypertension, diabetes mellitus, or hypercholesterolemia, systolic and diastolic blood pressure decreased in the period of at least half an hour after the sauna bath. In contrast, heart rate increased during this time [3]. After a few days of regular sauna bathing (10 sauna baths within 2 days), a reduction in total cholesterol and LDL cholesterol lasting up to several weeks occurs. After a few sauna sessions also an increase in HDL cholesterol and a decrease in triglycerides were observed [7]. The positive effect of sauna sessions on the lipid profile was similar to that obtained by moderate-intensity physical exercise [7].

Sauna use also has an effect on the hormonal metabolism of the body. Regular sauna sessions lead to an increase in norepinephrine (NA) and antidiuretic hormone (ADH) levels. This activates the Renin-Angiotensin-Aldosterone (RAA) system. Sauna bathing also increases the levels of growth hormone $(\mathrm{GH})$, prolactin (PRL) and beta-endorphin. Within a few hours after the session, hormone levels return to normal [8].

The following rapid cooling in cold water after the warm-up period causes a sudden drop-in heart rate to value similar to those before the warm-up began. According to studies on young healthy subjects, both systolic and diastolic blood pressure remain unchanged [9].

\section{Health benefits and risks associated with sauna bathing}

\subsection{Health benefits of different types of sauna bathing}

Sauna bathing may reduce the risk of respiratory diseases, treatment of chronic headache and skin diseases like psoriasis and urticaria [10,11]. The high temperature of the sauna bath also helps to eradicate dermatophytes [10]. High temperature also destroys pathogenic microorganisms in the upper respiratory tract, which can be effective in the treatment of some infectious diseases [12]. The studies also showed that sauna bathing is associated with lowered risk of cardiovascular diseases (CVD) [13], neurocognitive diseases like Alzheimer's disease and dementia [14]. Research conducted in Finland indicates that frequent sauna bathers are less likely to develop acute and chronic respiratory diseases. However, these studies were conducted on too low number of patients, which indicates the need for further research [15]. Studies have found sauna bathing to be associated with a better cardiovascular and circulatory function. The association between sauna bathing and risk of sudden cardiac death (SCD) and other cardiovascular diseases like fatal coronary heart disease (CHD) and fatal cardiovascular disease (CVD) is unknown. Sauna bathing leads to a reduction of blood pressure, which is caused by a reduced blood volume in the vascular bed due to intensive sweating. This results in an accelerated heart rate and increased cardiac output [16]. Heart rate may increase up to $100 / \mathrm{min}$ or even to $150 / \mathrm{min}$ during more intense sauna bathing session [16]. Therefore, people with orthostatic hypotension should be cautious when sauna bathing because of possible decrease in blood pressure after the sauna bathing. Frequent sauna bathing ( 2 times per week) improve heat tolerance during a lung period, increase vital capacity, minute ventilation and forced expiratory volume of the lungs [16]. Dry sauna bathing is safe even for patients after myocardial infarction and patients with stabile angina pectoris. In randomised clinical trials, sauna bathing was found to be a beneficial 
predictor in patients at increased risk of SCD, fatal CHD and all-cause mortality events. Due to the relatively small number of patients participating in clinical trials and the similar ethnicity (studies performed on the Finnish population), further studies carried out in other populations and other ethnic groups are needed.

Waon therapy consisting of sessions 3 times a week at $60^{\circ} \mathrm{C}$ has proven benefits in reducing mortality and risk of cardiovascular incidents in patients with NYHA class III and IV heart failure. Patients undergoing Waon therapy had significantly better results in the 6minute walk test $[17,18]$.

Sauna bathing also has a beneficial effect on reducing the risk of pneumonia. During a follow-up of more than 25 years, the hazard ratios (HR) for pneumonia adjusted for parameters such as diet, socioeconomic status, physical activity and C-reactive protein were 0.72 and 0.63 for patients taking sauna baths 2-3 and more than 4 times a week, in comparison to patients taking 1 or less. However, this study was conducted on a group limited to Caucasian men aged 42-61 years living in eastern Finland. This indicates the need for similar studies with patients from other ethnic groups [19].

\subsection{Health benefits associated with FIR therapy in infrared sauna}

Most of the molecular mechanisms of FIR's effects on the human body still remain unknown. Infrared sauna therapy may be associated with increased nitric oxide (NO) production in the vascular endothelium and with increased expression of endothelial NO synthase. In recent years, the application of FIR therapy in medicine has been systematically increasing and numerous studies have been conducted to determine the effect of infrared radiation on biological processes in the body. Due to the complex effects of radiation on the body, it has still not been possible to identify the exact mechanisms of the therapy. So far, the effects of FIR therapy have been confirmed for reducing oxidative stress, improving vascular endothelial function and inhibiting vasoconstriction after angioplasty (neointima hyperplasia) [20,21]. In a study published in 2004, Masuda et al. showed that FIR therapy reduces oxidative stress leading to atherosclerosis in patients with elevated risk factors for coronary artery disease [22]. The study of Huang et al. also showed a reduction in oxidative stress in patients with diabetes mellitus [23]. Animal studies have shown a beneficial effect of FIR in patients with chronic kidney disease (CKD) [20]. Due to the limited number of scientific studies on a larger number of patients, the mechanisms of action of FIR are still unknown which indicates the need for further research.

The use of infrared sauna in patients with rheumatoid arthritis and ankylosing spondylitis led to an improvement in general condition, a decrease in stiffness. During the 4week treatment an improvement of the clinical condition and well-being of the patients was observed. A short-term positive therapeutic effect was confirmed in clinical studies [24].

\subsection{Risks and contraindications of sauna bathing}

Patients who have had a heart attack or are chronically treated for heart failure are at increased risk of arrhythmia during sauna bathing. Among the most serious adverse consequences of sauna bathing are SCDs. Only 1-2\% of these occur within 24 hours of sauna bathing, and most of these are related to the consumption of ethyl alcohol [16]. For this reason, it is strictly forbidden to visit the sauna after drinking alcohol or drink alcohol inside the sauna. In the case of deaths occurring in the sauna, alcohol and existing cardiovascular diseases were the majority of the causes. Rarer causes of death include drowning, hypoxia, burns, carbon dioxide poisoning or other toxic substances [25]. Cardiac contraindications to 
the use of sauna bathing include congenital cyanotic heart diseases, aortic valve stenosis, congestive cardiomyopathy, poorly controlled hypertension. People with orthostatic hypotension and patients taking cardiovascular drugs should consult a doctor before using a sauna. Despite the relatively rare occurrence of life-threatening arrhythmias in public saunas, the presence of a defibrillator is recommended. There is also a significant risk of dehydration during sauna bathing, which is usually manifested by dizziness or visual disturbances. If people become dehydrated, it is recommended that they quickly replenish their fluids orally or intravenously [3].

In a study, sauna bathing twice a week for three months was shown to lead to impaired spermatogenesis and sperm quality, an effect that persists up to several months after sauna bathing is stopped [26]. This indicates the need for research into the effects of sauna bathing on male fertility in the reproductive age group.

\section{Discussion and conclusions}

Both the centuries-old traditions and scientific studies point to numerous health benefits of sauna bathing. However, most of these have been performed on limited populations living mainly in Sweden and Finland. This indicates a need for research in other populations and ethnic groups. It is also necessary to better understand the biological processes occurring in the body during a sauna session. This will allow the appropriate selection of sauna bathing parameters individually to the needs of the patient. Sauna bathing also has a positive impact on mental condition of visitors. Sauna bathing treatments can be an effective complement to the process of treatment and rehabilitation of patients with cardiovascular diseases, musculoskeletal diseases, respiratory tract diseases, skin diseases and many others. Due to its multiple benefits and relatively low cost, heat therapy can be successfully applied to numerous patients. However, it is always necessary to remember about the risk of exposure to high temperatures. The use of sauna in the case of patients with chronic diseases, especially cardiovascular diseases should be always preceded by consultation with a doctor.

\section{References:}

[1] Laukkanen JA, Laukkanen T, Kunutsor SK. Cardiovascular and Other Health Benefits of Sauna Bathing: A Review of the Evidence. Mayo Clin Proc 2018;93:1111-21. https://doi.org/10.1016/j.mayocp.2018.04.008.

[2] Pawłowski J, Pawłowska K, Bochyński R. Meaning of sauna bath in human body health training. Med Ogólna i Nauk o Zdrowiu 2015;21:282-8. https://doi.org/10.5604/20834543.1165354.

[3] Olsson B. [Medical aspects on sauna bathing]. Lakartidningen 2018;115.

[4] Vatansever F, Hamblin MR. Far infrared radiation (FIR): its biological effects and medical applications. Photonics Lasers Med 2012;4:255-66. https://doi.org/10.1515/plm-2012-0034.

[5] Mero A, Tornberg J, Mäntykoski M, Puurtinen R. Effects of far-infrared sauna bathing on recovery from strength and endurance training sessions in men. Springerplus 2015;4:321. https://doi.org/10.1186/s40064-015-1093-5.

[6] Pilch W, Szygula Z, Palka T, Pilch P, Cison T, Wiecha S, et al. Comparison of physiological reactions and physiological strain in healthy men under heat stress in dry and steam heat saunas. Biol Sport 2014;31:145-9. https://doi.org/10.5604/20831862.1099045.

[7] Gryka D, Pilch W, Szarek M, Szygula Z, Tota $Ł$. The effect of sauna bathing on lipid 
profile in young, physically active, male subjects. Int J Occup Med Environ Health 2014;27:608-18. https://doi.org/10.2478/s13382-014-0281-9.

[8] Laukkanen JA, Laukkanen T. Sauna bathing and systemic inflammation. Eur J Epidemiol 2018;33:351-3. https://doi.org/10.1007/s10654-017-0335-y.

[9] Kauppinen K. Sauna, shower, and ice water immersion. Physiological responses to brief exposures to heat, cool, and cold. Part II. Circulation. Arctic Med Res 1989;48:64-74.

[10] Hannuksela M, Väänänen A. The sauna, skin and skin diseases. Ann Clin Res 1988;20:276-8.

[11] Kanji G, Weatherall M, Peter R, Purdie G, Page R. Efficacy of regular sauna bathing for chronic tension-type headache: a randomized controlled study. J Altern Complement Med 2015;21:103-9. https://doi.org/10.1089/acm.2013.0466.

[12] Cohen M. Turning up the heat on COVID-19: heat as a therapeutic intervention. F1000Research 2020;9:292. https://doi.org/10.12688/f1000research.23299.2.

[13] Laukkanen T, Khan H, Zaccardi F, Laukkanen JA. Association between sauna bathing and fatal cardiovascular and all-cause mortality events. JAMA Intern Med 2015;175:542-8. https://doi.org/10.1001/jamainternmed.2014.8187.

[14] Laukkanen T, Kunutsor S, Kauhanen J, Laukkanen JA. Sauna bathing is inversely associated with dementia and Alzheimer's disease in middle-aged Finnish men. Age Ageing 2017;46:245-9. https://doi.org/10.1093/ageing/afw212.

[15] Kunutsor SK, Laukkanen T, Laukkanen JA. Sauna bathing reduces the risk of respiratory diseases: a long-term prospective cohort study. Eur J Epidemiol 2017;32:1107-11. https://doi.org/10.1007/S10654-017-0311-6.

[16] Laukkanen T, Khan H, Zaccardi F, Laukkanen JA. Association between sauna bathing and fatal cardiovascular and all-cause mortality events. JAMA Intern Med 2015;175:542-8. https://doi.org/10.1001/jamainternmed.2014.8187.

[17] Tei C, Imamura T, Kinugawa K, Inoue T, Masuyama T, Inoue H, et al. Waon Therapy for Managing Chronic Heart Failure - Results From a Multicenter Prospective Randomized WAON-CHF Study. Circ J 2016;80:827-34. https://doi.org/10.1253/circj.CJ-16-0051.

[18] Kihara T, Miyata M, Fukudome T, Ikeda Y, Shinsato T, Kubozono T, et al. Waon therapy improves the prognosis of patients with chronic heart failure. J Cardiol 2009;53:214-8. https://doi.org/10.1016/j.jjcc.2008.11.005.

[19] Kunutsor SK, Laukkanen T, Laukkanen JA. Frequent sauna bathing may reduce the risk of pneumonia in middle-aged Caucasian men: The KIHD prospective cohort study. Respir Med 2017;132:161-3. https://doi.org/10.1016/J.RMED.2017.10.018.

[20] Shui S, Wang X, Chiang JY, Zheng L. Far-infrared therapy for cardiovascular, autoimmune, and other chronic health problems: A systematic review. Exp Biol Med 2015;240:1257. https://doi.org/10.1177/1535370215573391.

[21] Kipshidze N, Nikolaychik V, Muckerheidi M, Keelan MH, Chekanov V, Maternowski $\mathrm{M}$, et al. Effect of short pulsed nonablative infrared laser irradiation on vascular cells in vitro and neointimal hyperplasia in a rabbit balloon injury model. Circulation 2001;104:1850-5. https://doi.org/10.1161/hc3901.096101.

[22] Masuda A, Miyata M, Kihara T, Minagoe S, Tei C. Repeated sauna therapy reduces urinary 8-epi-prostaglandin F(2alpha). Jpn Heart J 2004;45:297-303. https://doi.org/10.1536/jhj.45.297.

[23] Huang P-H, Chen J-W, Lin C-P, Chen Y-H, Wang C-H, Leu H-B, et al. Far infra-red therapy promotes ischemia-induced angiogenesis in diabetic mice and restores high glucose-suppressed endothelial progenitor cell functions. Cardiovasc Diabetol 2012;11:99. https://doi.org/10.1186/1475-2840-11-99. 
[24] Oosterveld FGJ, Rasker JJ, Floors M, Landkroon R, van Rennes B, Zwijnenberg J, et al. Infrared sauna in patients with rheumatoid arthritis and ankylosing spondylitis. Clin Rheumatol 2009;28:29-34. https://doi.org/10.1007/s10067-008-0977-y.

[25] Rodhe A, Eriksson A. Sauna deaths in Sweden, 1992-2003. Am J Forensic Med Pathol 2008;29:27-31. https://doi.org/10.1097/PAF.0b013e318145ae05.

[26] Garolla A, Torino M, Sartini B, Cosci I, Patassini C, Carraro U, et al. Seminal and molecular evidence that sauna exposure affects human spermatogenesis. Hum Reprod 2013;28:877-85. https://doi.org/10.1093/humrep/det020. 